\title{
ACUTE MYOPIA AS OCULAR MANIFESTATION OF SYSTEMIC LUPUS ERYTHEMATOSUS
}

\author{
Rodrigo Barbosa de Azevedo ${ }^{1}$, Priscila Dourado Evangelista ${ }^{1, \star}$, Thalita do Nascimento Silva ${ }^{1}$ \\ 1.Hospital Geral Dr. César Cals, Fortaleza (CE), Brazil. \\ *Corresponding author: prisdourado@yahoo.com.br
}

\section{BACKGROUND}

Systemic lupus erythematosus (SLE) is a multisystem autoimmune disease with heterogeneous clinical features. Ophthalmic manifestations, detected in approximately one-third of SLE patients, may be present at the onset or during the course of the disease, which can affect any part of the visual system and is usually indicative of disease activity. In this study, we reported a patient with SLE who presented ocular involvement and sudden development of myopia, which significantly improved with immunosuppression.

\section{CASE REPORT}

A 22-year-old female presented with complaints of 15 days of periorbital edema, blurred vision and significant reduction of visual acuity. There was no history of trauma or previous ocular pathologies. In addition, she referred with anorexia, abdominal pain, intermittent diarrhea with rectal bleeding, alopecia, malar rash with photosensitivity, oral ulcers, daily fever and polyarthralgia. Laboratory tests revealed hemoglobin: $8.5 \mathrm{~g} / \mathrm{dL}$, leukocytes: $4070 / \mathrm{mm}^{3}$ with lymphocytes: $710 / \mathrm{mm}^{3}$, platelets: 32000 , Coombs direct positive, C3: $31.5 \mathrm{mg} / \mathrm{dL}$ and C4: $3.23 \mathrm{mg} / \mathrm{dL}$ and proteinuria of $24 \mathrm{~h}$ of $1.32 \mathrm{~g}$. ANA, anti-DNA and anti-Sm positive, marking 12 criteria of Systemic Lupus International Collaborating Clinics (SLICC) 2012. Direct ophthalmoscopy showed signs of retinal vasculitis and optical coherence tomography (OCT) showed a subretinal hyporeflective space compatible with liquid provoking increased retinal thickness in the central macular area. A refractive disorder in the right eye (RE) -2.0 and in the left eye (LE) -1.75 and -0.25 at $130^{\circ}$, compatible with myopia, was also evidenced. Methylprednisolone $500 \mathrm{mg} /$ day for three consecutive days followed by cyclophosphamide at a monthly dose of $750 \mathrm{mg} / \mathrm{m}^{2}$ was performed. The body surface reached complete remission of disease after 6 months of treatment. In ophthalmologic reevaluation, there was evidence of complete improvement of the findings of vasculitis and retinal edema and the refractive disorder show complete improvement of myopia, with visual acuity of 20/20 in both eyes and +0.5 in RE and +0.5 in LE.

\section{CONCLUSION}

Acute myopia as the presenting sign in SLE is rare. In the databases PubMed and LILACS presents only 11 case reports with association of lupus and myopia. In our case, the appearance of the refractive disorder during high disease activity and its complete resolution with immunosuppression point to acute myopia as a probable ophthalmological manifestation secondary to SLE, which should be remembered by ophthalmologists and rheumatologists.

\section{KEYWORDS}

Myopia, Systemic lupus erythematosus, Retinal vasculitis. 